\title{
A note on the nonzero spectra of irreducible matrices
}

\author{
Shmuel Friedland \\ Department of Mathematics, Statistics and Computer Science \\ University of Illinois at Chicago \\ Chicago, Illinois 60607-7045, USA \\ E-mail: friedlan@uic.edu
}

\begin{abstract}
In this note we extend the necessary and sufficient conditions of BoyleHandleman 1991 and Kim-Ormes-Roush 2000 for a nonzero eigenvalue multiset of primitive matrices over $\mathbb{R}_{+}$and $\mathbf{Z}_{+}$, respectively, to irreducible matrices.
\end{abstract}

2010 Mathematics Subject Classification. 15A18, 15A29, 15A42, 15B36, $15 \mathrm{~B} 48$.

Key words. Inverse eigenvalue problems for nonnegative matrices.

\section{Introduction}

Denote by $\mathbb{R}^{n \times n} \supset \mathbb{R}_{+}^{n \times n}$ the algebra of real valued $n \times n$ matrices and the cone of $n \times n$ nonnegative matrices, respectively. For $A \in \mathbb{R}^{n \times n}$ denote by $\Lambda(A)=$ $\left\{\lambda_{1}(A), \ldots, \lambda_{n}(A)\right\}$ the eigenvalue multiset of $A$, i.e. $\operatorname{det}(z I-A)=\prod_{i=1}^{n}\left(z-\lambda_{i}(A)\right)$. An outstanding problem in matrix theory, called NIEP, is to characterize a multiset $\Lambda=\left\{\lambda_{1}, \ldots, \lambda_{n}\right\}$ which is an eigenvalue multiset of some $A \in \mathbb{R}^{n \times n}$. Denote by $\rho(\Lambda):=\max \{|\lambda|, \lambda \in \Lambda\}$, and by $\Lambda(r)$ all elements in $\Lambda$ satisfying $|\lambda|=r \geq 0$. For $\lambda \in \Lambda$ denote by $m(\lambda) \in \mathbb{N}$ the multiplicity of $\lambda$ in $\Lambda$. The obvious necessary conditions for $\Lambda=\Lambda(A)$ for some $A \in \mathbb{R}_{+}^{n \times n}$ are the trace conditions:

$$
s_{k}(\Lambda):=\sum_{i=1}^{n} \lambda_{i}^{k} \geq 0 \text { for } k=1, \ldots,
$$

since $s_{k}(\Lambda(A))=\operatorname{tr} A^{k}$. The following theorem is deduced straightforward from $[2$, Thm 2]. (See $\S 2$.)

Theorem 1.1 Let $\Lambda=\left\{\lambda_{1}, \ldots, \lambda_{n}\right\}$ be a multiset of complex numbers. Assume that the inequalities in (1.1) hold except for a finite number values of $k$. Then

1. $\bar{\Lambda}=\Lambda$.

2. $\rho(\Lambda) \in \Lambda$.

3. $m(\rho(\Lambda)) \geq m(\lambda)$ for all $\lambda \in \Lambda(\rho(\Lambda))$.

4. Assume that $\rho(\Lambda)>0$ and let $\left\{\lambda_{1}, \ldots, \lambda_{p}\right\}$ be all distinct elements of $\Lambda$ such that $\left|\lambda_{i}\right|=\rho(\Lambda)$ and $m\left(\lambda_{i}\right)=m(\rho(\Lambda))$ for $i=1, \ldots, p$. Then $\zeta \Lambda(\rho(\Lambda))=$ $\Lambda(\rho(\Lambda))$ for $\zeta=e^{\frac{2 \pi \sqrt{-1}}{p}}$. 
By considering the diagonal elements of $A^{k}$ and comparing them with the diagonal elements of $A^{k m}$ Loewy and London added the following additional necessary conditions [7].

Theorem 1.2 Let $\Lambda=\left\{\lambda_{1}, \ldots, \lambda_{n}\right\}$ be an eigenvalue multiset of some $A \in$ $\mathbb{R}_{+}^{n \times n}$. Then in addition to the inequalities (1.1) the following inequalities hold.

$$
s_{k m}(\Lambda) \geq \frac{1}{n^{k-1}}\left(s_{m}(\Lambda)\right)^{k} \text { for } m, k-1=1, \ldots
$$

In particular,

$$
\text { if } s_{m}(\Lambda)>0 \text { then } s_{k m}(\Lambda)>0 \text { for } k=2, \ldots
$$

The inequalities (1.1) and (1.2) imply that $\Lambda$ is an eigenvalue multiset of some $A \in \mathbb{R}_{+}^{n \times n}$ in the following cases: $n=3 ; n=4$ and $\Lambda$ is a multiset of real numbers. For $n=4$ and nonreal $\Lambda=\left\{\lambda_{1}, \lambda_{2}, \lambda_{3}, \lambda_{4}\right\}$ the conditions (1.1) and (1.2) are not sufficient [7]. The necessary and sufficient conditions are given in [9]. The inequality $n s_{4}(\Lambda) \geq\left(s_{2}(\Lambda)\right)^{2}$ in $(1.2)$ can be improved to $(n-1) s_{4}(\Lambda) \geq\left(s_{2}(\Lambda)\right)^{2}$ if $s_{1}(\Lambda)=0$ and $n$ is odd [8].

Definition 1.3 A multiset $\Lambda=\left\{\lambda_{1}, \ldots, \lambda_{n}\right\} \subset \mathbb{C}$ is called a Frobenius multiset if the following conditions hold.

1. $\bar{\Lambda}=\Lambda$.

2. $\rho(\Lambda) \in \Lambda$.

3. $m(\lambda)=1$ for each $\lambda \in \Lambda(\rho(\Lambda))$.

4. Assume that $\# \Lambda(\rho(\Lambda))=p$. Then $\zeta \Lambda=\Lambda$ for $\zeta=e^{\frac{2 \pi \sqrt{-1}}{p}}$.

The Frobenius theorem for irreducible $A \in \mathbb{R}_{+}^{n \times n}$, i.e. $(I+A)^{n-1}$ is a positive matrix, claims that $\rho(\Lambda(A))>0$ and $\Lambda(A)$ is a Frobenius set. In particular, an irreducible $A \in \mathbb{R}_{+}^{n \times n}$ is primitive, i.e. $A^{(n-1)^{2}+1}$ is a positive matrix, if and only if $\Lambda(\rho(\Lambda))=\{\rho(\Lambda)\}$, see $[3$, XII. $\S 5]$ and $[4, \S 8.5 .9]$.

We say that a multiset $\Lambda=\left\{\lambda_{1}, \ldots, \lambda_{n}\right\}$, where $\lambda_{i} \neq 0$ for $i=1, \ldots, n$, is a nonzero eigenvalue multiset of a nonnegative matrix if there exists an integer $N \geq n$ and $A \in \mathbb{R}_{+}^{N \times N}$, such that $\Lambda$ is obtained from $\Lambda(A)$ by removing all zero eigenvalues. The following remarkable theorem was proved by Boyle and Handelman [1]. Namely, a multiset $\Lambda \subset \mathbb{C} \backslash\{0\}$ is a nonzero spectrum of a nonnegative primitive matrix if and only if $\Lambda(\rho(\Lambda))=\{\rho(\Lambda)\}$, and the inequalities (1.1) and (1.3) hold. See the recent proof of Thomas Laffey [6] of a simplified version of this result. The aim of this note is to extend the theorem of Boyle-Handelman to a nonzero eigenvalue multiset of nonnegative irreducible matrices.

Theorem 1.4 Let $\Lambda$ be a multiset of nonzero complex numbers. Then $\Lambda$ is a nonzero eigenvalue multiset of a nonnegative irreducible matrix if and only if $\Lambda$ is a Frobenius set, and (1.1) and (1.3) hold.

Similarly, we extend the results of Kim, Ormes and Roush [5] to a nonzero eigenvalue multiset of nonnegative irreducible matrices with integer entries. 


\section{Proofs of Theorems 1.1 and 1.4}

Proof of Theorem 1.1. For $\Lambda=\{0, \ldots, 0\}$ the theorem is trivial. Assume that $\rho(\Lambda)>0$. Consider the function

$$
f_{\Lambda}(z)=\sum_{i=1}^{n} \frac{1}{1-\lambda_{i} z}=\sum_{k=0}^{\infty} s_{k}(\Lambda) z^{k} .
$$

Assume that $s_{k}(\Lambda) \geq 0$ for $k>N$. Then by subtracting a polynomial $P(z)$ of degree $N$ at most, we deduce that $f_{0}(z):=f(z)-P(z)$ has real nonnegative MacLaurin coefficients. So $\overline{f_{0}(\bar{z})}=f(z)$. Hence $\bar{\Lambda}=\Lambda$. The radius of convergence of this series is $R\left(f_{\Lambda}\right)=\frac{1}{\rho(\Lambda)}$. The principal part of $f$ is $f_{1}:=\sum_{i,\left|\lambda_{i}\right|=\rho(\Lambda)} \frac{1}{1-\lambda_{i} z}$. So $\pi\left(f_{\Lambda}(z)\right)=\left\{\left(\lambda_{1}, m\left(\lambda_{1}\right), 1\right), \ldots,\left(\lambda_{q}, m\left(\lambda_{q}\right), 1\right)\right\}$, where $\lambda_{1}, \ldots, \lambda_{q}$ are all pairwise distinct elements of $\Lambda(\rho(\Lambda))$, see [2, Dfn. 1]. Then parts 2-4 follow from [2, Thm. 2].

Proof of Theorem 1.4. Assume first that $\Lambda$ is a nonzero eigenvalue multiset of a nonnegative irreducible matrix. The Frobenius theorem yields that $\Lambda$ has to be a Frobenius set, and (1.1) and (1.3) hold. Assume now that $\Lambda$ is a Frobenius set, and (1.1) and (1.3) hold. In view of the Boyle-Handelman theorem it is enough to consider the case

$$
\Lambda(\rho(\Lambda))=\left\{\rho(\Lambda), \zeta \rho(\Lambda), \ldots, \zeta^{p-1} \rho(\Lambda)\right\}, \text { for } \zeta=e^{\frac{2 \pi \sqrt{-1}}{p}} \text { and } 1<p \in \mathbb{N} .
$$

Observe first that $s_{k}(\Lambda)=0$ if $p \nmid \chi k$. Let $\phi: \mathbb{C} \rightarrow \mathbb{C}$ be the map $z \mapsto z^{p}$. Since $\zeta \Lambda=\Lambda$, it follows that for $z \in \Lambda$ with multiplicity $m(z)$ the multiplicity of $z^{p}$ in $\phi(\Lambda)$ is $p m(z)$. Hence $\phi(\Lambda)$ is a union of $p$ copies of a Frobenius set $\Lambda_{1}$, where $\rho\left(\Lambda_{1}\right)=$ $\rho(\Lambda)^{p}$ and $\Lambda_{1}\left(\rho\left(\Lambda_{1}\right)\right)=\left\{\rho\left(\Lambda_{1}\right)\right\}$. Moreover $s_{k p}(\Lambda)=p s_{k}\left(\Lambda_{1}\right)$. Hence $\Lambda_{1}$ satisfies the assumptions of the Boyle-Handelman theorem. Thus there exists a primitive matrix $B \in \mathbb{R}_{+}^{M \times M}$ whose nonzero eigenvalue multiset is $\Lambda_{1}$. Let $A=\left[A_{i j}\right]_{i=j=1}^{p}$ be the following nonnegative matrix of order $p M$.

$$
A=\left[\begin{array}{cccccc}
0_{n \times n} & I_{n} & 0_{n \times n} & 0_{n \times n} & \ldots & 0_{n \times n} \\
0_{n \times n} & 0_{n \times n} & I_{n} & 0_{n \times n} & \ldots & 0_{n \times n} \\
\vdots & \vdots & \vdots & \vdots & \vdots & \vdots \\
0_{n \times n} & 0_{n \times n} & 0_{n \times n} & 0_{n \times n} & \ldots & I_{n} \\
B & 0_{n \times n} & 0_{n \times n} & 0_{n \times n} & \ldots & 0_{n \times n}
\end{array}\right]
$$

Then $A$ is irreducible and the nonzero part of eigenvalue multiset $\Lambda(A)$ is $\Lambda$.

\section{An extension of Kim-Ormes-Roush theorem}

In this section we give necessary and sufficient conditions on a multiset $\Lambda$ of nonzero complex number to be a nonzero eigenvalue multiset of a nonnegative irreducible matrix with integer entries. Recall the Möbius function $\mu: \mathbb{N} \rightarrow\{-1,0,1\}$. First $\mu(1)=1$. Assume that $n>1$. If $n$ is not square free, i.e. $n$ is divisible by $l^{2}$ for some positive integer $l>1$, then $\mu(n)=0$. If $n>1$ is square free, let $\omega(n)$ be the number of distinct primes that divide $n$. Then $\mu(n)=(-1)^{\omega(n)}$. The following theorem is a generalization of the Kim-Ormes-Roush theorem [5]. 
Theorem 3.1 Let $\Lambda$ be a multiset of nonzero complex numbers. Then $\Lambda$ is a nonzero eigenvalue multiset of a nonnegative irreducible matrix with integer entries if and only if the following conditions hold.

1. $\Lambda$ is a Frobenius set.

2. The coefficients of the polynomial $\prod_{\lambda \in \Lambda}(z-\lambda)$ are integers.

3. $t_{k}(\Lambda):=\sum_{d \mid k} \mu\left(\frac{k}{d}\right) s_{d}(\Lambda) \geq 0$ for all $k \in \mathbb{N}$.

The case $\Lambda(\rho(\Lambda))=\{\rho(\Lambda)\}$ is the Kim-Ormes-Roush theorem.

Proof. Assume that $\Lambda$ is a nonzero spectrum of a nonnegative irreducible matrix with integer entries, i.e. $A \in \mathbf{Z}_{+}^{N \times N}$. Then part 1 follows from the Frobenius theorem. Since $\operatorname{det}(z I-A)$ has integer coefficients we deduce part 2. It is known that $t_{k}(\Lambda)=t_{k}(\Lambda(A))$ is the number of minimal loops of length $k$ in the directed multigraph induced by $A$, see [1]. Hence part 3 holds.

Suppose that $\Lambda$ satisfies $1-3$. In view of the Kim-Ormes-Roush theorem it is enough to assume the case (2.1). We now use the notations and the arguments of the proof of Theorem 1.4. First $s_{k}(\Lambda)=0$ if $p \not k$. Second $\prod_{\lambda \in \Lambda}(z-\lambda)=\prod_{\kappa \in \Lambda_{1}}\left(z^{p}-\kappa\right)$. Hence $\prod_{\kappa \in \Lambda_{1}}(z-\kappa)$ has integer coefficients. A straightforward calculation shows that $t_{p k}(\Lambda)=p t_{k}\left(\Lambda_{1}\right)$. Hence $t_{k}\left(\Lambda_{1}\right) \geq 0$. Kim-Ormes-Roush theorem yields the existence of $B \in \mathbf{Z}_{+}^{M \times M}$ such that $\Lambda_{1}$ is the nonzero eigenvalue multiset of $B$. Hence $\Lambda$ is the nonzero eigenvalue set of $A \in \mathbf{Z}_{+}^{p M \times p M}$ given by (2.2).

\section{References}

[1] M. Boyle and D. Handelman, The spectra of nonnegative matrices via symbolic dynamics, Annals of Math. 133 (1991), 249-316.

[2] S. Friedland, On inverse problem for nonnegative and eventually nonnegative matrices, Israel J. Math. 29 (1978), 43-60.

[3] F.R. Gantmacher, The Theory of Matrices, Vol. I and II, Chelsea Publ. Co., New York 1959.

[4] R.A. Horn and C.R. Johnson, Matrix Analysis, Cambridge Univ. Press, New York 1988.

[5] K.H. Kim, N.S. Ormes and F.W. Roush, The spectra of nonnegative integer matrices via formal power series, J. Amer. Math. Soc. 13 (2000), 773-806.

[6] T.J. Laffey, A constructive version of the Boyle-Handelman theorem on the spectra of nonnegative matrices, arXiv:1005.0929.

[7] R. Loewy and D. London, A note on an inverse problem for nonnegative matrices, Linear Multilin. Algebra 6 (1978) 83-90.

[8] T. Laffey and E. Meehan, A refinement of an inequality of Johnson, Loewy and London on nonnegative matrices and some applications, Electron. J. Linear Algebra 3 (1998), 119-128 
[9] J. Torre-Mayo, M.R. Abril-Raymundo, E. Alarcia-Estvez, C. Marijun and M. Pisonero, The nonnegative inverse eigenvalue problem from the coefficients of the characteristic polynomial. EBL digraphs, Linear Algebra Appl. 426 (2007), no. 2-3, 729-773. 